\title{
Fan-Shaped Complete Block on Helical Tomotherapy for Esophageal Cancer: A Phantom Study
}

\author{
Chiu-Han Chang, ${ }^{1,2}$ Greta S. P. Mok, ${ }^{3}$ Pei-Wei Shueng, ${ }^{2}$ Hsin-Pei Yeh, ${ }^{2}$ An-Cheng Shiau, \\ Hui-Ju Tien, ${ }^{2}$ Chi-Ta Lin, ${ }^{2}$ and Tung-Hsin $\mathrm{Wu}^{1}$ \\ ${ }^{1}$ Department of Biomedical Imaging and Radiological Sciences, National Yang Ming University, Taipei 112, Taiwan \\ ${ }^{2}$ Division of Radiation Oncology, Department of Radiology, Far Eastern Memorial Hospital, New Taipei City 220, Taiwan \\ ${ }^{3}$ Biomedical Imaging Laboratory, Department of Electrical and Computer Engineering, Faculty of Science and Technology, \\ University of Macau, Macau \\ ${ }^{4}$ Department of Radiation Oncology, Koo Foundation Sun Yat-Sen Cancer Center, Taipei 112, Taiwan
}

Correspondence should be addressed to Greta S. P. Mok; gretamok@umac.mo and Tung-Hsin Wu; tung@ym.edu.tw

Received 10 November 2014; Revised 13 January 2015; Accepted 14 January 2015

Academic Editor: Xin-yuan Guan

Copyright (C) 2015 Chiu-Han Chang et al. This is an open access article distributed under the Creative Commons Attribution License, which permits unrestricted use, distribution, and reproduction in any medium, provided the original work is properly cited.

Radiation pneumonitis (RP) is a common complication for radiotherapy of esophageal cancer and is associated with the low dose irradiated lung volume. This study aims to reduce the mean lung dose (MLD) and the relative lung volume at $20 \mathrm{~Gy}\left(V_{20}\right)$ and at low dose region using various designs of the fan-shaped complete block (FSCB) in helical tomotherapy. Hypothetical esophageal tumor was delineated on an anthropomorphic phantom. The FSCB was defined as the fan-shaped radiation restricted area located in both lungs. Seven treatment plans were performed with nonblock design and FSCB with different fan angles, that is, from $90^{\circ}$ to $140^{\circ}$, with increment of $10^{\circ}$. The homogeneous index, conformation number, MLD, and the relative lung volume receiving more than 5 , 10,15 , and $20 \mathrm{~Gy}\left(V_{5}, V_{10}, V_{15}\right.$, and $\left.V_{20}\right)$ were determined for each treatment scheme. There was a substantial reduction in the MLD, $V_{5}, V_{10}, V_{15}$, and $V_{20}$ when using different types of FSCB as compared to the nonblock design. The reduction of $V_{20}, V_{15}, V_{10}$, and $V_{5}$ was $6.3 \%-8.6 \%, 16 \%-23 \%, 42 \%-57 \%$, and $42 \%-66 \%$ for FSCB $90^{\circ}-140^{\circ}$, respectively. The use of FSCB in helical tomotherapy is a promising method to reduce the MLD, $V_{20}$, and relative lung volume in low dose region, especially in $V_{5}$ and $V_{10}$ for esophageal cancer.

\section{Introduction}

Radiotherapy is an important component in multimodality treatment for patients with esophageal cancer. According to the Radiation Therapy Oncology Group (RTOG) 85-01 trial, for nonsurgical treatment, the standard therapy for patients with localized esophageal carcinoma is radiotherapy to $50 \mathrm{~Gy}$ plus concurrent chemotherapy for better control of local tumor and fewer distal metastases. According to the National Comprehensive Cancer Network (NCCN Ver. I.2013) guideline, factors including the area at risk for microscopic disease, setup uncertainties, and respiratory and swallow motion should be considered in the treatment planning. Thus, planning target volume (PTV) of esophageal cancer on the longitudinal direction is much longer than the gross tumor volume (GTV). However, several radiosensitive organs at risk (OARs) such as spinal cord, heart, lungs, liver, and kidneys that surrounded the PTV must be considered in the treatment planning to prevent potential complications. Sample complications include radiation pneumonitis (RP) and fibrosis in the lungs, pericardial effusion and myocardial ischemia in the heart, and myelitis in the spinal cord. Among them, RP is one of the most dose-limiting toxicities and acute complication for thoracic radiotherapy of esophageal cancer [1-5].

Asakura et al. [6] found all lung dose volume histogram (DVH) parameters significantly associated with grade 2 or higher RP and they determined the optimal threshold of relative lung volume $\left(V_{5}-V_{50}\right)$ to predict symptomatic RP. Another study suggested that lung volume receiving low dose 
related more to the incidence of RP as compared to the lung volume receiving high dose [7]. Wang et al. [2] ensured that the incidence of postoperative pulmonary complications reduced when an adequate volume of lung was spared of radiation.

Helical tomotherapy (HT) (Tomotherapy Inc., Madison, WI) is a modality for delivering intensity-modulated radiation therapy (IMRT) treatments using a rotating linear accelerator mounted on a continuously moving slip ring gantry in synchrony with the couch motion. Imageguided pretreatment alignment is feasible through megavoltage computed tomography (CT) scan to verify the tumor position. Compared to traditional IMRT or 3-dimensional conformal radiotherapy (3DCRT), a higher degree of dose conformity and homogeneity in the target can be achieved for HT due to larger number of degrees of freedom in beam arrangement during gantry rotations to fit the lesion slice by slice, while sparing the adjacent OARs at the same time [8-13]. Chandra et al. [13] suggested that when more beams at different directions crossfire at the tumor, the volume of normal tissue with low-dose exposure may be increased and the lateral beams that irradiated a larger lung volume should be avoided. However, standard HT using 51 gantry angles through $360^{\circ}$ in esophageal cancer led to extensive low-dose distribution in both lungs. Although directional and complete blocks of radiation beams are feasible in HT, currently there are no clear guidelines and systematic evaluations in reduction of the lung volume with low-dose exposure for treating esophageal cancer with HT.

In this study, we proposed to reduce the lung volume with low-dose exposure in HT for esophageal cancer by "blocking" the radiation beams, that is, closing the MLC, at certain angles especially in the lateral direction to spare the exposure of OARs. The directional block was defined as avoiding only incoming radiation beams while the complete block was defined as restricting the radiation beams to enter and exit certain volume $[3,14]$. The degree of blocking was further quantified by the fan-shaped complete block (FSCB) to indicate the fan angles of beam blocking.

\section{Material and Methods}

An anthropomorphic body phantom (ATOM 701; CIRS, Norfolk, VA) was scanned under a CT simulator (Discovery, GE, USA) in this study. The CT slice thickness was $2.5 \mathrm{~mm}$ for the entire thorax. We delineated the normal OARs and a hypothetical esophageal tumor, that is, the GTV, located at the middle esophagus of the thorax with size of $102.4 \mathrm{~cm}^{3}$ and longitudinal length of $12.5 \mathrm{~cm}$ on every CT slice using the Pinnacle treatment planning system (Version 7.6; Philips Medical Systems North America, Andover, MA, USA). The clinical target volume (CTV) was defined as the GTV extended $0.5 \mathrm{~cm}$ radially and $4 \mathrm{~cm}$ superiorly and inferiorly to cover region with subclinical disease. The PTV was defined as the CTV plus $0.8 \mathrm{~cm}$ margin in 3 dimensions for daily setup uncertainties and internal movement, including respiration and swallowing motion. The size of PTV is $497.73 \mathrm{~cm}^{3}$ with longitudinal length of $22.1 \mathrm{~cm}$ from apex of lung to the top of

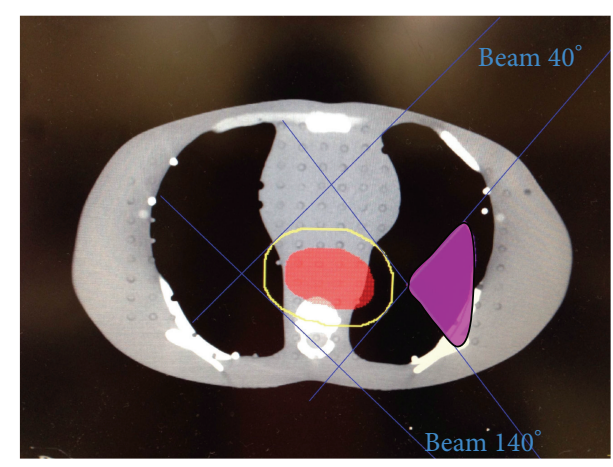

FIGURE 1: The fan-shaped block of FSCB $100^{\circ}$ (pink area) was defined by the intersection of beam angle of $40^{\circ}$ and $140^{\circ}$. The PTV was shown in red and the blue line defined the block auxiliary structure which was sufficient to cover $1.5 \mathrm{~cm}$ expanded from the PTV (yellow circle).

liver. Other outlined structures included spinal cord, heart, right lung, left lung, and the virtual block.

We designed 6 virtual FSCBs to form a fan-shaped block of radiation in both lungs with fan angles from $90^{\circ}$ to $140^{\circ}$, each with increment of $10^{\circ}$. In order to avoid insufficient dose coverage at the peripheral PTV after using the FSCBs, the block auxiliary structure was sufficient to cover region expanding $1.5 \mathrm{~cm}$ from the PTV margin in 3 dimensions (Figure 1). For our standard phantom, we determined the 2beam angles to achieve the fan-shaped block by the following equation for the left lung:

$$
\begin{gathered}
\text { Beam angle } \# 1=\frac{(180-\text { fan angle })}{2}, \\
\text { Beam angle } \# 2=180-\frac{(180-\text { fan angle })}{2} .
\end{gathered}
$$

Thus, Figure 1 showed that the FSCB $100^{\circ}$ was defined as the intersection of the beam with beam angle of $40^{\circ}$ and $140^{\circ}$ in the left lung. Similarly, the FSCB $100^{\circ}$ in right lung was delineated with the beam angles of $320^{\circ}$ and $220^{\circ}$.

After contouring all structures, the CT images were transferred to the HT planning system (Version 3.2.2.35 Tomotherapy Inc., Madison, WI). A prescription dose of $50.4 \mathrm{~Gy}$ in 28 fractions was defined for the $95 \%$ PTV. The parameters for treatment planning were field width of $2.5 \mathrm{~cm}$, pitch of $0.287[15,16]$, and modulation factor of 3.0 with normal resolution mode. During the dose optimization process the dose constraints were adjusted to obtain adequate and homogeneous target volume coverage while minimizing dose in heart, spinal cord, and both lungs. The dose constraints of normal OARs were based on the RTOG 1010 and the related studies $[2,4-7]$ and they are summarized as follows: the maximum dose was $<45 \mathrm{~Gy}$ for the spinal cord; the mean dose of heart was $<34 \mathrm{~Gy}$ and $V_{40}$ of the heart was $<50 \%$; the mean lung dose (MLD) must be $<20 \mathrm{~Gy}$; and percent volume of the organ receiving more than a threshold dose, that is, 20, 15, 10, and $5 \mathrm{~Gy}\left(V_{20}, V_{15}, V_{10}\right.$, and $\left.V_{5}\right)$ for lungs, was expected to be $<20 \%, 30 \%, 50 \%$, and $55 \%$, respectively. 


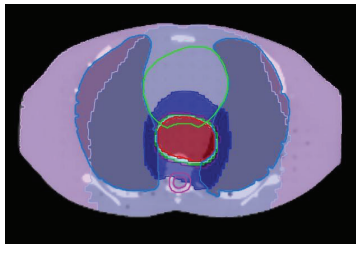

Non block

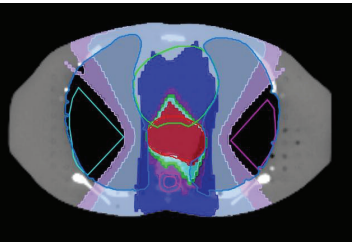

FSCB $90^{\circ}$

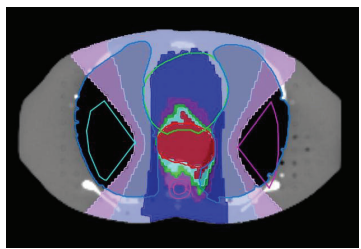

$\operatorname{FSCB} 100^{\circ}$

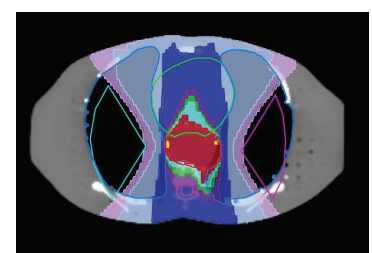

FSCB $110^{\circ}$

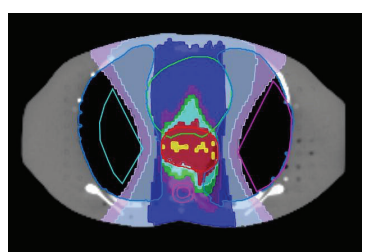

FSCB $120^{\circ}$

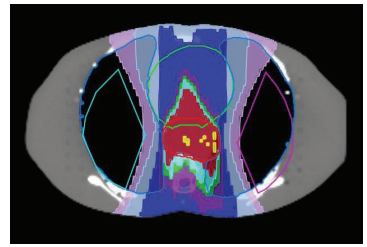

FSCB $130^{\circ}$

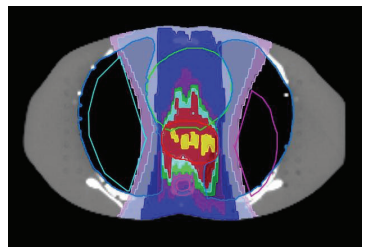

FSCB $140^{\circ}$

Figure 2: The dose distribution for nonblock and FSCB $90^{\circ}$ to $140^{\circ}$ designs.

This study evaluated various FSCBs with different fan angles and nonblock design and investigated the correlation between the reduction of low-dose lung volume and different beam angles of FSCB. For PTV, the homogeneous index (HI) $[17,18]$ was defined as the ratio of minimum dose received in $5 \%$ and $95 \%$ of the PTV:

$$
\mathrm{HI}=\frac{D_{5}}{D_{95}} .
$$

A HI value closer to 1 indicates better dose homogeneity. The conformation number $(\mathrm{CN})$ proposed by Riet et al. $[19,20]$ takes irradiation of both target volume and healthy tissues into account:

$$
\mathrm{CN}=\frac{\mathrm{TV}_{\mathrm{RI}}}{\mathrm{TV}} \times \frac{\mathrm{TV}_{\mathrm{RI}}}{V_{\mathrm{RI}}}
$$

where $\mathrm{TV}_{\mathrm{RI}}=$ target volume covered by the reference isodose, $\mathrm{TV}=$ target volume, and $V_{\mathrm{RI}}=$ volume with the reference isodose. A CN value closer to 1 indicates good target conformity and coverage.

Several dosimetric parameters including MLD, $V_{20}, V_{15}$, $V_{10}, V_{5}$ for lungs and $\mathrm{HI}$ and $\mathrm{CN}$ were evaluated for different fan angles of FSCB.

\section{Result}

The dose distribution for the seven treatment plans is shown in Figure 2. For the nonblock treatment planning, low-dose region with $5 \mathrm{~Gy}$ and $10 \mathrm{~Gy}$ spread among both lungs. For FSCB with larger fan angles, more lung volume could be protected. However, the prescribed dose would be extended to the anterior and posterior regions of the PTV. Also, the conformity and homogeneity of PTV would be degraded and the dose on spinal cord and heart was higher as compared to the nonblock design.

Different quantitative dose indices for the OARs and PTV were shown in Table 1 . In the nonblock design, the $\mathrm{CN}$ and the HI were $~ 1.0$, indicating homogenous dose distribution and idea conformation within the target volume. However, its $V_{10}$ and $V_{5}$ were $>75 \%$, indicating most of lung volume received low-dose exposure. The MLD and $V_{20}$ from different FSCB designs were less than half of dose compared to the nonblock design. The $V_{5}, V_{10}$, and $V_{15}$ for the lungs were also substantially lower for the FSCB designs (Figure 3), with simultaneous increase of the maximum dose of spinal cord, mean dose, and $V_{40}$ of the heart. Their $\mathrm{HI}$ and $\mathrm{CN}$ for the PTV degraded correspondingly.

\section{Discussion}

Previous studies showed that HT provides dosimetric merits in target coverage and homogeneity, as well as better dose distribution and sharp dose gradient in reducing complication probability in OARs [10]. Our study showed that the $\mathrm{HI}$ and $\mathrm{CN}$ were good; OARs such as spinal cord and heart received relatively low dose in the nonblock treatment planning, probably due to unconstrained irradiation beam angle in rotational dose delivery. With the use of FSCB, the irradiation beam angle of HT would be restricted to protect the fan-shaped area in the lungs. Hence, when the fan angle of the FSCB is larger, a relatively larger lung volume will be protected. The reduction of lung volume irradiated in lowdose region, MLD and $V_{20}$ from FSCB can potentially lead to 
TABLE 1: Quantitative dose indices for lungs, spinal cord, heart, and tumor for treatment plan of nonblock and various FSCB designs.

\begin{tabular}{|c|c|c|c|c|c|c|c|}
\hline & Nonblock & FSCB $90^{\circ}$ & FSCB $100^{\circ}$ & FSCB $110^{\circ}$ & FSCB $120^{\circ}$ & FSCB $130^{\circ}$ & FSCB $140^{\circ}$ \\
\hline \multicolumn{8}{|l|}{ Lungs } \\
\hline MLD (Gy) & $14.53 \pm 0.94$ & $9.38 \pm 0.26$ & $9.06 \pm 0.11$ & $8.73 \pm 0.09$ & $8.12 \pm 0.04$ & $7.33 \pm 0.32$ & $7.05 \pm 0.04$ \\
\hline$V_{20}(\%)$ & $18.04 \pm 0.91$ & $11.76 \pm 1.46$ & $11.42 \pm 0.47$ & $10.70 \pm 0.99$ & $10.57 \pm 0.80$ & $10.28 \pm 1.02$ & $9.38 \pm 0.13$ \\
\hline$V_{15}(\%)$ & $36.84 \pm 0.22$ & $21.11 \pm 2.03$ & $20.10 \pm 0.77$ & $18.85 \pm 0.30$ & $17.27 \pm 0.37$ & $14.49 \pm 1.86$ & $14.13 \pm 0.27$ \\
\hline$V_{10}(\%)$ & $77.19 \pm 4.72$ & $34.34 \pm 1.03$ & $32.24 \pm 0.16$ & $30.31 \pm 3.71$ & $28.14 \pm 1.48$ & $21.10 \pm 2.40$ & $20.68 \pm 0.26$ \\
\hline$V_{5}(\%)$ & $97.03 \pm 0.12$ & $54.07 \pm 0.63$ & $52.41 \pm 0.80$ & $46.74 \pm 1.35$ & $41.50 \pm 0.68$ & $33.41 \pm 1.32$ & $30.79 \pm 0.19$ \\
\hline \multicolumn{8}{|l|}{ Spinal cord } \\
\hline maximum dose (Gy) & $33.57 \pm 0.57$ & $41.64 \pm 0.79$ & $41.87 \pm 0.64$ & $42.86 \pm 0.45$ & $43.41 \pm 0.15$ & $44.02 \pm 0.14$ & $44.45 \pm 0.10$ \\
\hline \multicolumn{8}{|l|}{ Heart } \\
\hline mean dose (Gy) & $20.93 \pm 0.50$ & $29.15 \pm 0.83$ & $29.27 \pm 0.36$ & $31.03 \pm 0.41$ & $31.50 \pm 1.11$ & $32.11 \pm 0.94$ & $32.41 \pm 0.37$ \\
\hline$V_{40}(\%)$ & $6.72 \pm 0.03$ & $13.97 \pm 1.22$ & $15.07 \pm 0.94$ & $17.60 \pm 0.44$ & $19.02 \pm 3.77$ & $23.26 \pm 1.59$ & $25.78 \pm 0.50$ \\
\hline \multicolumn{8}{|l|}{ PTV } \\
\hline $\mathrm{CN}$ & $0.91 \pm 0.00$ & $0.82 \pm 0.02$ & $0.82 \pm 0.01$ & $0.82 \pm 0.00$ & $0.77 \pm 0.02$ & $0.75 \pm 0.03$ & $0.71 \pm 0.01$ \\
\hline HI & $1.02 \pm 0.00$ & $1.04 \pm 0.00$ & $1.04 \pm 0.00$ & $1.06 \pm 0.00$ & $1.07 \pm 0.00$ & $1.07 \pm 0.01$ & $1.07 \pm 0.00$ \\
\hline
\end{tabular}

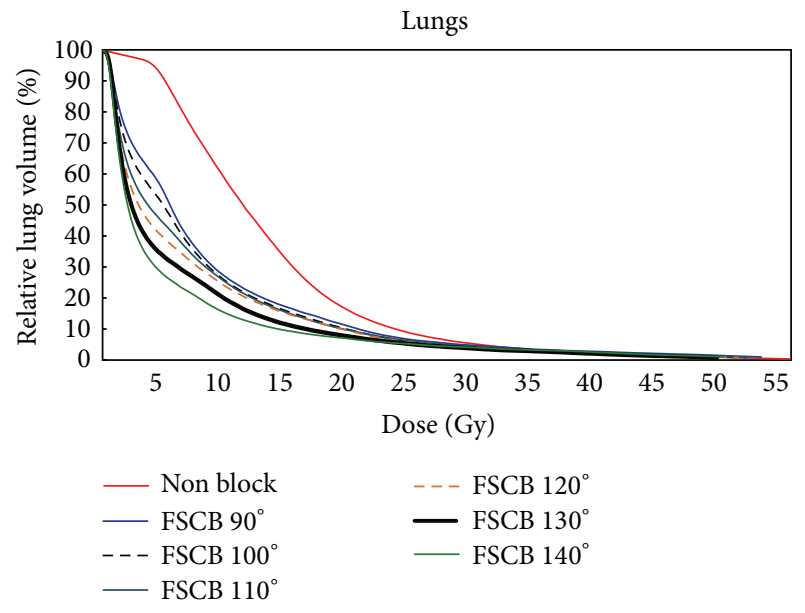

FIGURE 3: Cumulative DVHs of lung comparing nonblock and FSCB with various fan angles.

reduced incident of $\mathrm{RP}$ as studies have found that the volume of lung spared from doses of $5 \mathrm{~Gy}$ (VS5) was the major factor associated with pulmonary complications [2]. Also, RP has been shown to be associated with $V_{20}>20 \%$ [21] and MLD > 13.6 Gy [22]. Thus, it is important to reduce the MLD, $V_{20}$ and low-dose volume in both lungs for rotational beam delivery.

However, the maximum dose of spinal cord, the mean dose, and $V_{40}$ of heart increased and the $\mathrm{CN}$ and $\mathrm{HI}$ became worse with larger fan angle of the FSCB. That was probably due to the fact that the main direction for beam delivery is either anterior-posterior (AP) or posterior-anterior (PA) position. The spinal cord is a critical organ and its maximum dose was set to $45 \mathrm{~Gy}$ in the treatment planning. The heart received more radiation dose in FSCB designs, that is, 9$12 \mathrm{~Gy}$ and $7-19 \%$ increase in mean dose and $V_{40}$, respectively. However, these doses were still lower than the conventional AP:PA fields followed by off-cord oblique field technique in esophageal treatment planning.
This phantom study showed a quantitative dosimetric comparison between traditional nonblock and FSBC designs for HT. However, the optimal radiation treatment plan for esophageal cancer is actually patient-specific as it is highly dependent on the actual tumor volume. Smaller fan angle will be used for a larger tumor and less lung volume can be protected. For example, only smaller fan angles of FSCB, that is, $90^{\circ}$ and $100^{\circ}$, are recommended for elliptical PTV which includes gross lymph node and elective nodal regions to assure sufficient peripheral coverage of PTV. However, it will lead to more lung dose as compared to larger fan angles. For PTV only includes gross tumor volume, larger fan angles of $110^{\circ}-140^{\circ}$ are suggested. When the PTV is not located on the body midline, asymmetric fan angle of FSCB in right and left lung should be considered. For example, when the PTV covers the gastroesophageal junction which is near the left lung, the fan angle of FSCB in the left lung would be smaller than that in the right lung. We can also adjust the location of beam angles \#1 and \#2; that is, beam angle \#1 in left lung can be placed at the posterior lung to protect more left lung volume, if we prefer the same fan angle for both lungs. The size of the tumor volume also influences the FSCB design. Otherwise, the location of the virtual fan shape structure can be adjusted to protect more lung volume. The shape of the chest also affects the total lung volume and the fan angle of FSCB, with larger fan angle $\left(120^{\circ}-140^{\circ}\right)$ for thick chest and smaller fan angle $\left(90^{\circ}-110^{\circ}\right)$ for thin chest. In clinical practice, all aforementioned factors should be considered and proper fan angle can be decided for optimal tradeoffs among protected lung volume, irradiated dose to OARs and PTV dose conformity and homogeneity. With the use of FSCB, the treatment time will increases accordingly due to prescription dose deliver in restricted beam angle, that is, $14-25.6$ mins for FSBC $90^{\circ}-140^{\circ}$, while it is 8 mins for nonblock design.

\section{Conclusion}

This phantom study showed that HT with FSCB can reduce MLD, $V_{20}$, and low-dose lung volume, especially in $V_{5}$ and $V_{10}$ 
for esophageal tumor. And the maximum dose of spinal cord and mean dose of heart were increased because of the incident beams from anterior and posterior direction of the helical rotation. For the FSCB plans, CN and HI in PTV are slightly worse and treatment time is longer than nonblock design plan. The evaluated FSCB fan angles of $90^{\circ}-140^{\circ}$ can cover most clinical cases and the dose reduction increased for larger fan angle. Since MLD, $V_{20}$, and low-dose lung volume are reduced with FSCB designed in $\mathrm{HT}$ and also highly associated with RP, FSCB designed in HT is a promising protective method for reducing RP.

\author{
Abbreviations \\ GTV: Gross tumor volume \\ CTV: Clinical target volume \\ PTV: Planning target volume \\ OAR: Organ at risk \\ RP: $\quad$ Radiation pneumonitis \\ FSCB: Fan-shaped complete block \\ DVH: Dose volume histogram \\ MLD: Mean lung dose \\ HI: Homogeneous index \\ $\mathrm{CN}$ : Conformation number \\ HT: Helical tomotherapy \\ IMRT: Intensity-modulated radiation therapy \\ NCCN: National Comprehensive Cancer Network \\ RTOG: Radiation Therapy Oncology Group \\ MLC: Multileaf collimator.
}

\section{Conflict of Interests}

The authors declare that there is no conflict of interests regarding the publication of this paper.

\section{Authors' Contribution}

Greta S. P. Mok and Tung-Hsin Wu contributed equally to this work.

\section{Acknowledgments}

This study was financially supported by the Ministry of Science and Technology of Taiwan (MOST 103-2314-B-195001-MY3) and Multi-Year Research Grant of University of Macau, Macau (no. MYRG185(Y3-L3)-FST11-MSP).

\section{References}

[1] S. Tanabe, M. Myojin, S. Shimizu et al., "Dose-volume analysis for respiratory toxicity in intrathoracic esophageal cancer patients treated with definitive chemoradiotherapy using extended fields," Journal of Radiation Research, vol. 54, no. 6, pp. 1085-1094, 2013.

[2] S.-L. Wang, Z. Liao, A. A. Vaporciyan et al., "Investigation of clinical and dosimetric factors associated with postoperative pulmonary complications in esophageal cancer patients treated with concurrent chemoradiotherapy followed by surgery," International Journal of Radiation Oncology, Biology, Physics, vol. 64, no. 3, pp. 692-699, 2006.

[3] C.-T. Lin, A.-C. Shiau, H.-J. Tien, H.-P. Yeh, P.-W. Shueng, and C.-H. Hsieh, "An attempted substitute study of total skin electron therapy technique by using helical photon tomotherapy with helical irradiation of the total skin treatment: a phantom result," BioMed Research International, vol. 2013, Article ID 108794, 7 pages, 2013.

[4] F.-M. Hsu, Y.-C. Lee, J.-M. Lee et al., "Association of clinical and dosimetric factors with postoperative pulmonary complications in esophageal cancer patients receiving intensity-modulated radiation therapy and concurrent chemotherapy followed by thoracic esophagectomy," Annals of Surgical Oncology, vol. 16, no. 6, pp. 1669-1677, 2009.

[5] J. M. Schallenkamp, R. C. Miller, D. H. Brinkmann, T. Foote, and Y. I. Garces, "Incidence of radiation pneumonitis after thoracic irradiation: dose-volume correlates," International Journal of Radiation Oncology Biology Physics, vol. 67, no. 2, pp. 410-416, 2007.

[6] H. Asakura, T. Hashimoto, S. Zenda et al., "Analysis of dosevolume histogram parameters for radiation pneumonitis after definitive concurrent chemoradiotherapy for esophageal cancer," Radiotherapy and Oncology, vol. 95, no. 2, pp. 240-244, 2010.

[7] H. K. Lee, A. A. Vaporciyan, J. D. Cox et al., "Postoperative pulmonary complications after preoperative chemoradiation for esophageal carcinoma: correlation with pulmonary dosevolume histogram parameters," International Journal of Radiation Oncology Biology Physics, vol. 57, no. 5, pp. 1317-1322, 2003.

[8] Y.-J. Chen, A. Liu, C. Han et al., "Helical tomotherapy for radiotherapy in esophageal cancer: a preferred plan with better conformal target coverage and more homogeneous dose distribution," Medical Dosimetry, vol. 32, no. 3, pp. 166-171, 2007.

[9] M. Van Vulpen, C. Field, C. P. J. Raaijmakers et al., "Comparing step-and-shoot IMRT with dynamic helical tomotherapy IMRT plans for head-and-neck cancer," International Journal of Radiation Oncology Biology Physics, vol. 62, no. 5, pp. 1535-1539, 2005.

[10] S. Martin, J. Z. Chen, A. Rashid Dar, and S. Yartsev, "Dosimetric comparison of helical tomotherapy, RapidArc, and a novel IMRT \& Arc technique for esophageal carcinoma," Radiotherapy and Oncology, vol. 101, no. 3, pp. 431-437, 2011.

[11] C. Han, A. Liu, T. E. Schultheiss, R. D. Pezner, Y.-J. Chen, and J. Y. C. Wong, "Dosimetric comparisons of helical tomotherapy treatment plans and step-and-shoot intensity-modulated radiosurgery treatment plans in intracranial stereotactic radiosurgery," International Journal of Radiation Oncology Biology Physics, vol. 65, no. 2, pp. 608-616, 2006.

[12] N. P. Nguyen, S. P. Krafft, V. Vinh-Hung et al., "Feasibility of tomotherapy to reduce normal lung and cardiac toxicity for distal esophageal cancer compared to three-dimensional radiotherapy," Radiotherapy and Oncology, vol. 101, no. 3, pp. 438-442, 2011.

[13] A. Chandra, T. M. Guerrero, H. H. Liu et al., "Feasibility of using intensity-modulated radiotherapy to improve lung sparing in treatment planning for distal esophageal cancer," Radiotherapy and Oncology, vol. 77, no. 3, pp. 247-253, 2005.

[14] T. Reynders, K. Tournel, P. de Coninck et al., "Dosimetric assessment of static and helical TomoTherapy in the clinical implementation of breast cancer treatments," Radiotherapy and Oncology, vol. 93, no. 1, pp. 71-79, 2009. 
[15] M. W. Kissick, J. Fenwick, J. A. James et al., "The helical tomotherapy thread effect," Medical Physics, vol. 32, no. 5, pp. 14141423, 2005.

[16] J. Balog, G. Olivera, and J. Kapatoes, "Clinical helical tomotherapy commissioning dosimetry," Medical Physics, vol. 30, no. 12, pp. 3097-3106, 2003.

[17] T. Kataria, K. Sharma, V. Subramani, K. P. Karrthick, and S. S. Bisht, "Homogeneity Index: an objective tool for assessment of conformal radiation treatments," Journal of Medical Physics, vol. 37, no. 4, pp. 207-213, 2012.

[18] M. Yoon, S. Y. Park, D. Shin et al., "A new homogeneity index based on statistical analysis of the dose-volume histogram," Journal of Applied Clinical Medical Physics, vol. 8, no. 2, pp. 9-17, 2007.

[19] A. V. Riet, A. C. A. Mak, M. A. Moerland, L. H. Elders, and W. van der Zee, "A conformation number to quantify the degree of conformality in brachytherapy and external beam irradiation: application to the prostate," International Journal of Radiation, Oncology, Biology, Physics, vol. 37, no. 3, pp. 731-736, 1997.

[20] L. Feuvret, G. Noël, J.-J. Mazeron, and P. Bey, "Conformity index: a review," International Journal of Radiation Oncology Biology Physics, vol. 64, no. 2, pp. 333-342, 2006.

[21] K. Tsujino, S. Hirota, M. Endo et al., "Predictive value of dose-volume histogram parameters for predicting radiation pneumonitis after concurrent chemoradiation for lung cancer," International Journal of Radiation Oncology Biology Physics, vol. 55, no. 1, pp. 110-115, 2003.

[22] J. S. A. Belderbos, W. D. Heemsbergen, K. De Jaeger, P. Baas, and J. V. Lebesque, "Final results of a phase I/II dose escalation trial in non-small-cell lung cancer using three-dimensional conformal radiotherapy," International Journal of Radiation Oncology Biology Physics, vol. 66, no. 1, pp. 126-134, 2006. 


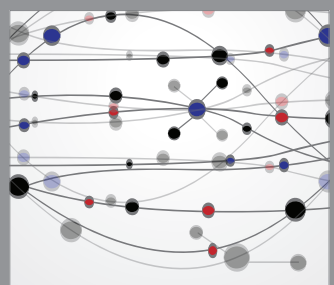

The Scientific World Journal
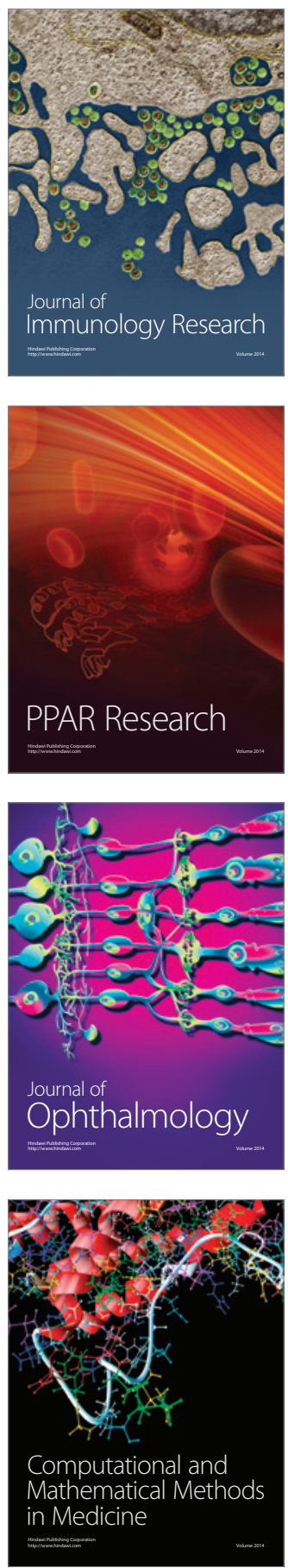

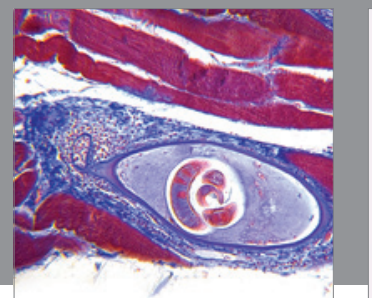

Gastroenterology

Research and Practice
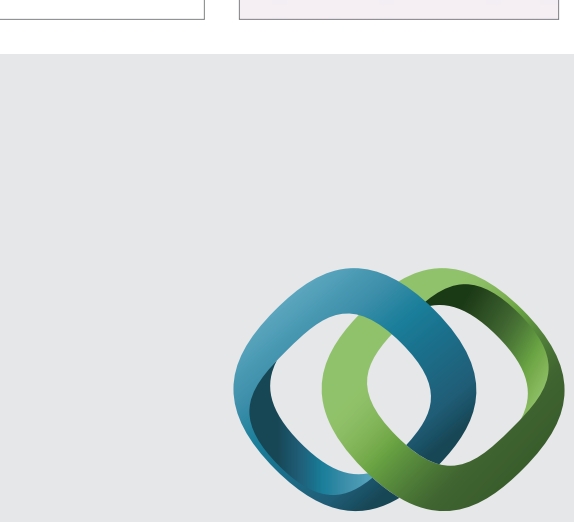

\section{Hindawi}

Submit your manuscripts at

http://www.hindawi.com
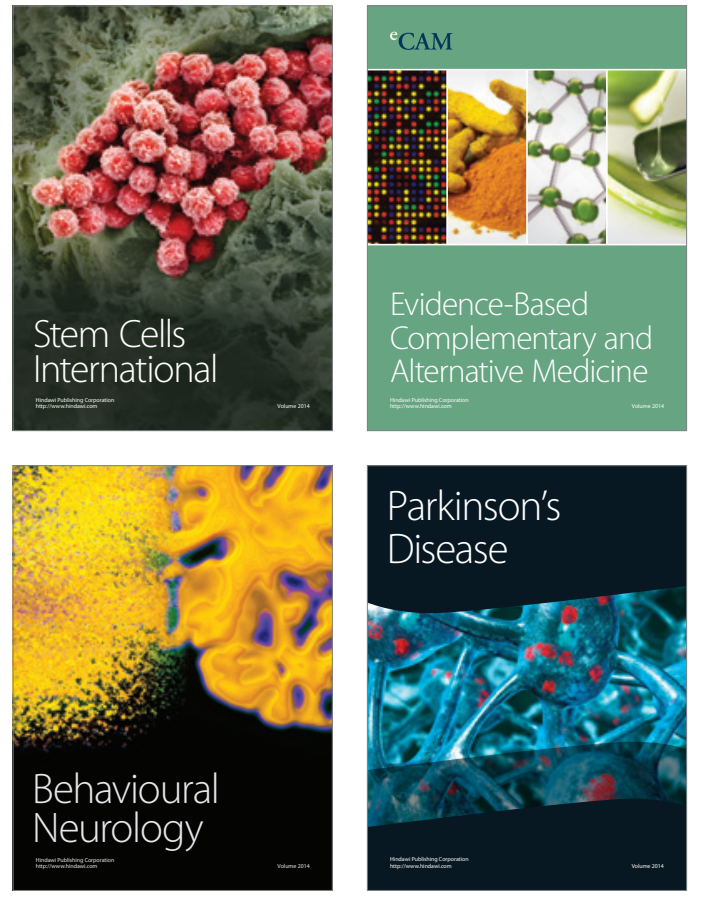
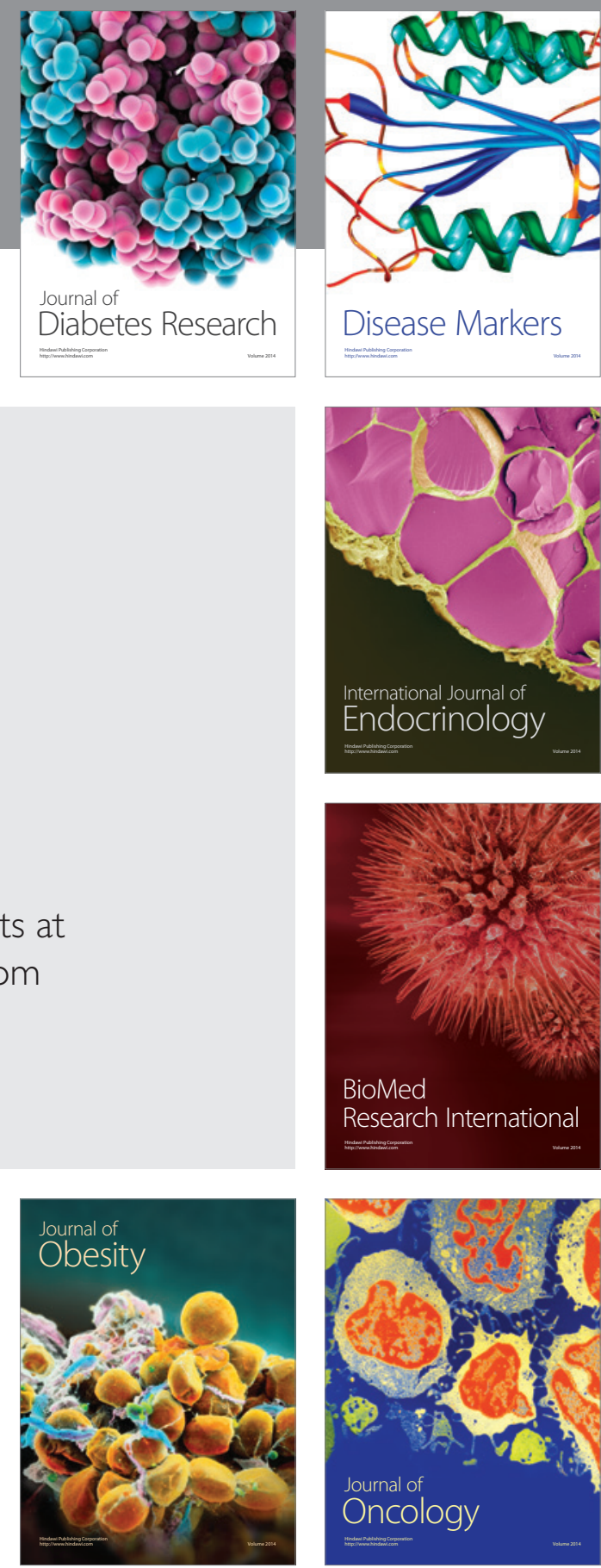

Disease Markers
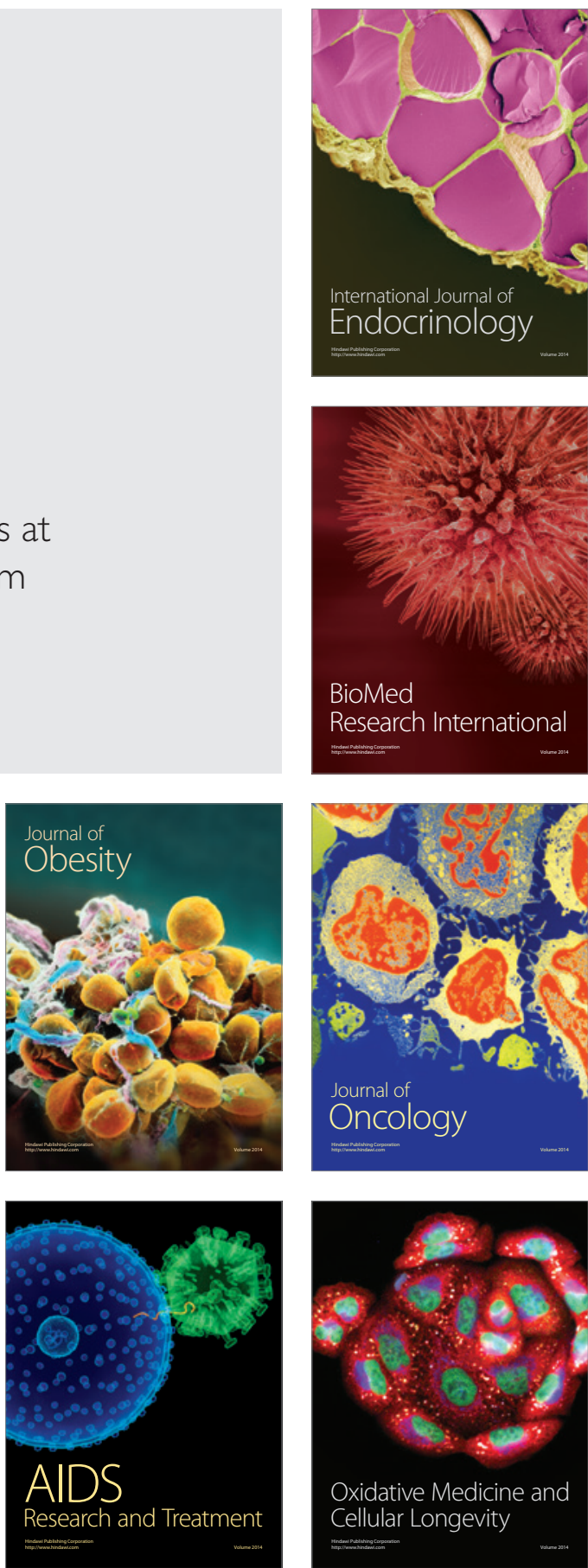